\title{
A Fast Optical Method for the Determination of Liquid Levels in Microplates
}

\author{
Kerstin Thurow, ${ }^{1}$ Norbert Stoll, ${ }^{2}$ and Kai Ritterbusch ${ }^{1}$ \\ ${ }^{1}$ Center for Life Science Automation, University of Rostock, F.-Barnewitz-Straße 1, 18119 Rostock, Germany \\ ${ }^{2}$ Institute of Automation, University of Rostock, R.-Wagner-Straße 31, 18119 Rostock, Germany
}

Correspondence should be addressed to Kerstin Thurow, kerstin.thurow@celisca.de

Received 14 January 2011; Accepted 24 April 2011

Academic Editor: Bernd Hitzmann

Copyright ( $) 2011$ Kerstin Thurow et al. This is an open access article distributed under the Creative Commons Attribution License, which permits unrestricted use, distribution, and reproduction in any medium, provided the original work is properly cited.

\begin{abstract}
Parallel liquid handling systems are widely used in different applications of life sciences. In order to avoid false positive or negative results which lead to higher costs due to the replication of the experiments it is necessary to monitor the process and success of liquid delivery. An easy method for the determination of the liquid levels in microplates has been developed and evaluated. The optical method bases on the measurement of the liquid level using CCD cameras followed by special algorithms for the evaluation and visualization of the measured data. The proposed method was tested in changing environmental lighting for two different liquids. These tests confirm our approach towards optical liquid level determination for smallest volumes in microplates and also show the challenges regarding environmental lighting and different physical properties of fluids.
\end{abstract}

\section{Introduction}

Expensive and time-consuming processes have forced an increase in efficiency in the research industry; this, as well as the increasing need for documentation, introduces the problem of pipetting robots operating blind. The operator provides the reagents and substances for the system to work with according to a preprogrammed procedure, leaving results in the form of value measurements at the end of the experiment-when identifying the root cause for outliers is difficult and corrective actions are no longer possible.

It may therefore make sense to introduce a pipetting monitoring system to monitor individual handling procedures. Pipetting problems are especially rife where extremely small samples need to be pipetted [1]. The pipetting monitoring system presented here is a low-priced, rapid, and space-saving solution towards detecting extremely small amounts in wells. The monitoring results are then added to the documentation or fed back into the control system to initialise a repeat pipetting procedure.

Detecting fluid fill levels plays a major role in many industrial specialities, so many methods of determining fill levels have been developed and reported on in various application areas. Weighing, pressure measurement on vessel bottoms, and ultrasound are a few examples of measurement principles that have been used $[2,3]$, while optical methods have also been used in a large number of applications, for example, where contact-free measurement is needed [4]. So far, there have not been any reports on using optical methods in determining fluid fill levels in lab automation systems, but a patent filed in 1989 did describe a mechanism for manual visual control on microtitre plates (MTPs) [5]. Using this method, MTPs to be examined are laid into the device that holds them at a predefined parallel distance to a patterned surface. The light reflected from the patterned surface is refracted through the fluid in the wells of transparent MTPs, allowing detection of fluid from distortions in the pattern or excess intensity compared to the empty wells. This method makes manual monitoring easy for the user, but it is still restricted to clear, colourless microtitre plates.

Currently, automated pipetting systems use capacitive or air pressure-sensitive pipettes to determine fill level [6], where the level is determined by detecting the change in capacity on contact between the tip of the pipette and the fluid or change in pressure as the pipette tip is dipped into the fluid. This capacitive measurement principle is restricted 
to fluids that conduct electricity, which may then have to be added to the actual sample; this restriction does not apply to the pressure principle, however.

Both systems measure fill levels using gap measurement and volume calculation based on the known geometry of the wells. However, the volumes that apply here amount to 0 $20 \mu \mathrm{l}$ for 96-well and $0-10 \mu \mathrm{l}$ for 384-well plates-the fluid still forms a droplet on the bottom of the well; classical fill-level measurement in these volume ranges will not yield usable results.

Using the methods described above, the pipettes would also need to keep to a minimum distance to the bottom of the well to avoid damage. Apart from that, small off-centre droplets in the well due to adhesion effects would remain undetectable.

Ultrasound sensors may be used in determining volume and also support contact-free measurement. There are sensors that are small enough to allow multiple well measurements [7]; the restrictions named above regarding the minimum gap from the pipette to the bottom of the plate or nondetection of off-centre droplets do not apply here.

\section{Optical Method towards Qualitative Fill-Level Detection in MTPs}

2.1. Requirements and Challenges. The principles named above have one particular drawback-measurement is timeconsuming, as each sensor can only monitor one well at a time. Capacitive measurement with an eight-tip pipette tool on a ninety-six-well plate would take around half a minute to complete and around two for 384 wells. Ultrasound sensors are faster, but, to be used for 96-well plates, thin sensors must be used that can be arranged in a row, where each well's width is $9 \mathrm{~mm}$. They have to be mounted on the robot above a labware position. Since most robots approach labware (to pick up and transport) from above, a conveyor must be used to transport the plate below the sensory equipment. These necessities make the solution difficult to install and expensive. Furthermore it needs a relatively large amount of space and would not be usable for 384-well plates. These fill-level detector systems could theoretically provide a way of monitoring individual pipetting steps, but the time taken would be considerably longer.

The optical pipette monitoring system developed would provide an adequate way of measuring each dosing step on several wells in parallel, supporting monitoring for 384 wells at very short times of less than ten seconds. Evaluation covers detecting droplets and subsequent estimation of droplet volume from the two-dimensional projection of the droplet on the image plane and the well-known geometry. This paper will describe the development and evaluation of the first step.

2.2. Selecting a Suitable Optical System. Camera systems and flatbed scanners are already used for reading barcodes in labs. Camera systems provide a fast imaging method at around a second, but they are very sensitive to ambient light; in addition, they photograph each well from a different angle depending on position. The speed benefit cannot outweigh the drawbacks for image processing, as monitoring pipettes requires an extremely high degree of accuracy (sensitivity and specificity).

Flatbed systems are an alternative, as they light each well directly from below, making the necessary image processing much easier. The Contact Image Sensor (CIS) system (ZTSA6, Ziath Ltd.) has a weak light source and depth of field; the weak light source renders the scanner sensitive to alterations in ambient lighting conditions, but the low depth of field presents an advantage in that only objects on the bottom of the well are in focus, making them easier to recognise. In contrast, Charge Coupled Device (CCD) flatbed scanners (Xtr-96, fluidX Ltd.) have a strong light source and greater depth of field. The plus and minus points of each sensor type can be weighed up depending on application-the software supports a variety of imaging systems.

2.3. System Setup. Various approaches towards implementing pipetting monitoring have been examined. The system was designed in order to integrate the hardware needed on the pipetting robot deck (Biomek FX, Beckman Coulter) and support a suitable interface to the control computer. In addition, a variety of coverings were tried out in order to determine and minimise the effects of ambient lighting. Additional software needs to be installed on the computer to be controlled by the control software. The software supports a variety of sensor types connected via USB, ensuring convenience and simplicity in installing the device.

2.4. Software. The image evaluation software, which was developed by the authors, is the heart of the measuring system; it can be used with a variety of imaging systems and can be configured for various plate types that may differ in bottom shape and material, and therefore also transparency. The image processing concept was inspired by cascade classifiers [8] since it was not initially possible to develop an algorithm that would be equally suitable for the various plate types, droplet sizes, and fluids. Cascade classifiers consist of a number of weak classifiers that combine to a single strong one.

Three algorithms (the weak classifiers; see Table 1) evaluate an image simultaneously; output is merged with an ADALINE (Adaptive Linear Neuron) to calculate the result [9]. Optimum weights exist to configure the system for each plate and device combination (see Figure 1). The weights are determined from training data using a simple least-squares approach.

Finally, a fixed threshold needs to be selected in order to use the system; the ideal threshold $t_{\mathrm{opt}}$ is determined from the training data as follows:

$$
t_{\mathrm{opt}}=\max [\mathrm{TP}(t)-\mathrm{FP}(t)]
$$

The a priori knowledge on the plate, imaging device, and pipetted fluids is used for other points as well. The system selects the settings required for the imaging method and the algorithm parameters by reference to plate-device combination. 
TABle 1: Procedure and parameters in the algorithms designed.

\begin{tabular}{|c|c|c|c|}
\hline Name & TemplateBased() & ReflexionPattern() & HoughCircles() \\
\hline $\begin{array}{l}\text { Computational } \\
\text { steps }\end{array}$ & $\begin{array}{l}\text { (i) Difference between image and } \\
\text { template } \\
\text { (ii) Thresholding (1) } \\
\text { (iii) Morphological opening }(2,3) \\
\text { (iv) Sum over pixel values equals blob size }\end{array}$ & $\begin{array}{l}\text { (i) Blurring } \\
\text { (ii) Edge detection with Sobel filter (1) } \\
\text { (iii) Calculation of statistical moments } \\
\text { (iv) Calculation of moment differences } \\
\text { between image and template }\end{array}$ & $\begin{array}{l}\text { (i) Laplacian-of-Gaussian filter (1) } \\
\text { (ii) Hough-transformation }(2,3) \\
\text { (iii) Calculation of the distance } \\
\text { between well tangent } \\
\text { and object tangent }\end{array}$ \\
\hline $\begin{array}{l}\text { Parameters to } \\
\text { adjust for } \\
\text { labware and } \\
\text { device }\end{array}$ & $\begin{array}{l}\text { (1) Threshold } \\
\text { (2) Size of the structuring element } \\
\text { (3) Iterations }\end{array}$ & (1) Filter order in $x$-and $y$-direction & $\begin{array}{l}\text { (1) Filter threshold } \\
\text { (2) Accumulator threshold } \\
\text { (3) Min/max Radius }\end{array}$ \\
\hline Result & Blob size & Differences in variance, skewness, excess & $\begin{array}{l}\text { Radius, distance between well } \\
\text { and object tangents }\end{array}$ \\
\hline
\end{tabular}

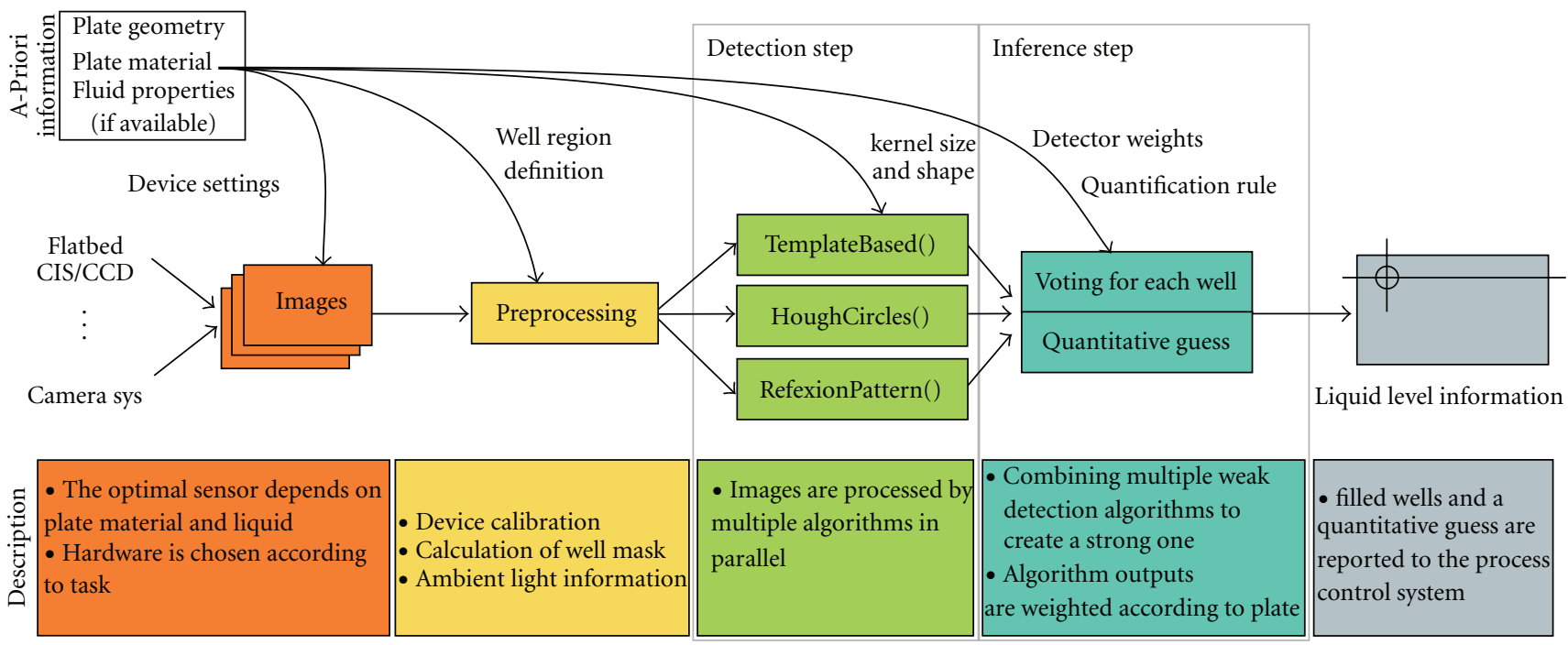

Figure 1: Program flow scheme.

2.5. Examination Approach. Each device was set up on the pipetting platform for testing. Fluids were dosed via the pipetting system. The plates were then transferred to the optical system; an image was taken of each with (CIS, camera) and without covering (CIS, CCD, camera). The camera system was not included for evaluation for the reasons stated in Section 2.2.

To generate more test data, the plates were used several times with accumulating amounts of substance inside. Two pipetting approaches were used for the examinations. For the 96-well plates a certain volume was dispensed to each of the 8 wells in one row whereas the following row was left empty ( 6 rows filled, 6 rows empty). For the 384-well plate a certain volume was dispensed to every second well. These approaches were selected in order to measure the droplets spread over the plate. There were also empty wells distributed across the plate for position effects to be detected as well.

Dosages of 1-40 $\mu \mathrm{l}$ were taken for the 96-well MTPs, but only $1-12 \mu \mathrm{l}$ max dosage for the 384-well plates due to the smaller well volume. Tighter steps were used for $1-8 \mu \mathrm{l}$ dosages to generate more test data. The algorithms developed were used afterwards on the data collected.
The tests were run on 96-well polystyrene (PS) and polypropylene (PP) plates with V-bottoms (V), a 96-well polystyrene plate with a flat bottom (F), anda 384-well plate polypropylene plate with a V-bottom. In the following, we will be referring to the plates by the above abbreviations. Images were taken in three to four runs each for four plates in eight to twelve volume steps in three types of setup-CCD, CIS with, and CIS without covering. In total, more than four hundred test images were taken.

2.6. Evaluation Method. A receiver operating characteristic (ROC) function describes the detector's behaviour depending on threshold selection setting its sensitivity [10]. If a low threshold is set, many true positives but more false alarms would be expected.

Efficacy was expressed as true positives (TPs) and false positives (FPs) for one selected threshold Table 3. Regardless of threshold, the area under the ROC curve is taken to measure its efficacy [10]. The ROC curve is a function with the threshold as its parameter. The criteria from Table 2 can be used in order to test for efficacy. 
TABLE 2: Evaluation criteria.

\begin{tabular}{ll}
\hline True positives & $\mathrm{TP}=$ Detections/Wells $\mathrm{sull}_{\text {fl }}$ \\
\hline False positives & $\mathrm{FP}=$ FalseAlarm $/$ Wells $\mathrm{smpty}_{\mathrm{e}}$ \\
\hline Area under curve & $\mathrm{AUC}=\int \mathrm{TP} d \mathrm{FP}$ \\
\hline
\end{tabular}
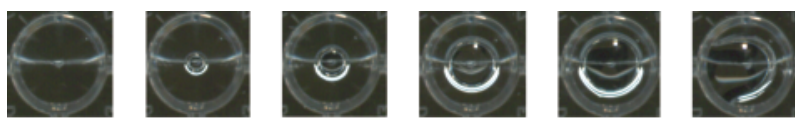

Figure 2: A growing drop, connecting to the wall between $24 \mu \mathrm{l}$ and $32 \mu \mathrm{l}$.

\section{Results}

Table 1 summarises the true and false positives for the plates examined for each device as a percentage. This table shows an example of one run with fixed parameters optimised for V-PP-96 plates and CIS system (without covering).

Wide-ranging examinations were performed on system sensitivity to various influences in order to gauge the usefulness of the system in real-life conditions.

3.1. Influence of Resolution on Efficacy. The tests were performed at a maximum resolution of $600 \mathrm{dpi}(0.042 \mathrm{~mm}$ per pixel). Comparative tests on lower resolutions of $300 \mathrm{dpi}$ ( $0.085 \mathrm{~mm}$ per pixel) also yielded good results. Lowering resolution in the volume ranges tested above $1 \mu \mathrm{l}$ showed no significant negative effect.

3.2. The System's Measurement Range. There are upper and lower volume limits for detecting droplets. The lower limit is subject to several factors, such as light distorting the image in the middle of V-bottom wells at the cylinder tip or V-tip, and the droplet needs to be larger than the bottom tip. For all plates the lower limit was found to be below $1 \mu \mathrm{l}$.

Figure 2 shows how droplets tend to stick to the walls of a well in recognisably increased numbers, when growing in size.

This reduces the TP rate for large volumes since the reflexions disappear and hence leads to the upper boundary of the measurement range. The ideal measuring range for 96-well plates was between 1 and $24 \mu \mathrm{l}$, while volumes of up to $10 \mu \mathrm{l}$ were recognisable in 384 -well plates. Figure 3 shows the degradation of measurement quality with two different plates.

3.3. Operation under Changing Lighting Conditions. The system would be used in a variety of conditions in real-life operation; ambient light reaching the plate varies depending on the system's location as well as the season and time of day, so the system would have to be tested for robustness with regard to light conditions. To test this, test images were taken in three different typical lighting situations as follows: (1) full direct sunlight on the device, (2) indirect daylight, and (3) no natural lighting-lab lighting.

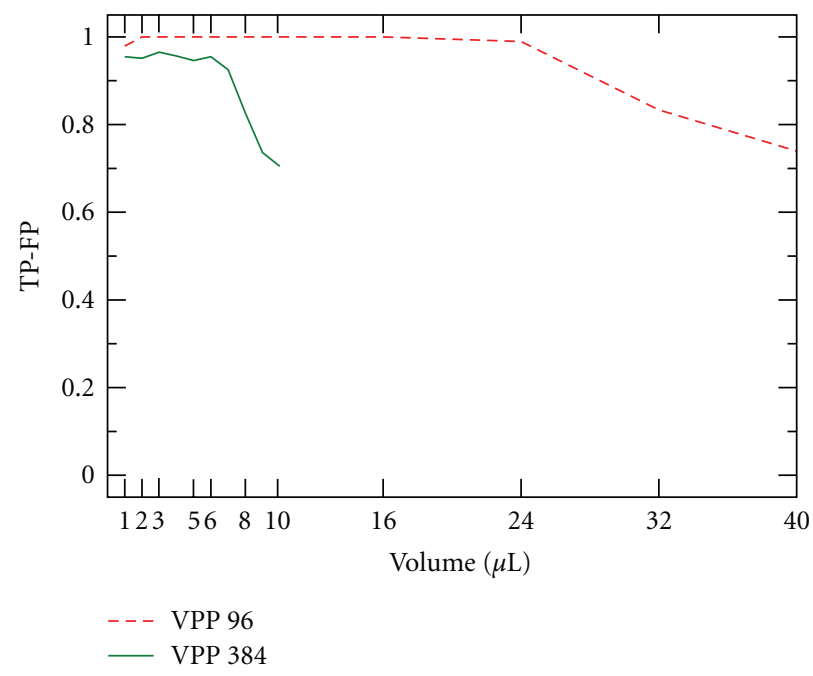

Figure 3: Degradation of measurement accuracy with growing sample volume.

Two images were taken for each run using the CIS sensor to test its particular sensitivity towards ambient light. Each plate was imaged once with and once without the covering (microtitre plate lid) in each ambient light setting and with every volume. The lid shields the plate from ambient light during the imaging process.

Figure 4 shows an example of how direct sunlight ruined the image. Similar overexposure also affected images made using the CCD sensor, albeit less strongly since the CCD sensor is less sensitive to ambient light. Overall, no images taken with the CCD sensor were affected by direct light such that they became useless.

The results for the CIS scanner with lid are represented by the hashed area in Figure 4 . The solid area includes results without the lid. The figure shows the improved relationship between true positives against false positives on the one hand and the smaller fluctuation range on the other, using the lid. The benefits of covering therefore consist of improved results and robustness to ambient light. The smaller fluctuation range is equivalent to a smaller area on the TP/FP plane. The bounded area $\triangle \mathrm{AUC}_{o}$ was 0.132 without covering compared to $\triangle \mathrm{AUC}_{l}$ at 0.018 with covering (lid) —only around $13.6 \%$.

The CCD sensor yielded more robust results; the difference in area $\triangle \mathrm{AUC}_{o, \mathrm{CCD}}$ was 0.034 for the CCD sensor, the same general range as with the CIS sensor with covering.

3.4. Influence of Sample Substance and Pipetting Technique. Substances to be dosed vary according to fluid properties. Fluids with varying levels of viscosity and surface tension are dosed using different pipetting techniques. If the cohesion effects between the fluid and pipette are too heavy, the droplets have to be placed onto the well using techniques such as Tip Touch. Viscosity also affects the way samples of a given volume spread across the well.

One test addressed functionality with filtered water and pure DMSO. The ideal pipetting technique for water was used for the droplets of the same shape to be placed 
TABLE 3: Results in percent.

\begin{tabular}{lcccccc}
\hline & \multicolumn{2}{c}{ CIS open } & & & CIS lid & \multicolumn{2}{c}{ CCD open } \\
\hline & TP & FP & TP & FP & TP & FP \\
\hline VPP384 & 99.5 & 0.0 & 96.1 & 0.0 & 90.2 & 9.0 \\
VPS96 & 97.8 & 0.0 & 86.7 & 0.0 & 96.6 & 0.4 \\
FPS96 & 88.7 & 1.0 & 86.3 & 5.4 & 72.6 & 3.0 \\
VPP384 & 94.2 & 7.5 & 96.5 & 4.2 & 95.4 & 0.6 \\
\hline
\end{tabular}

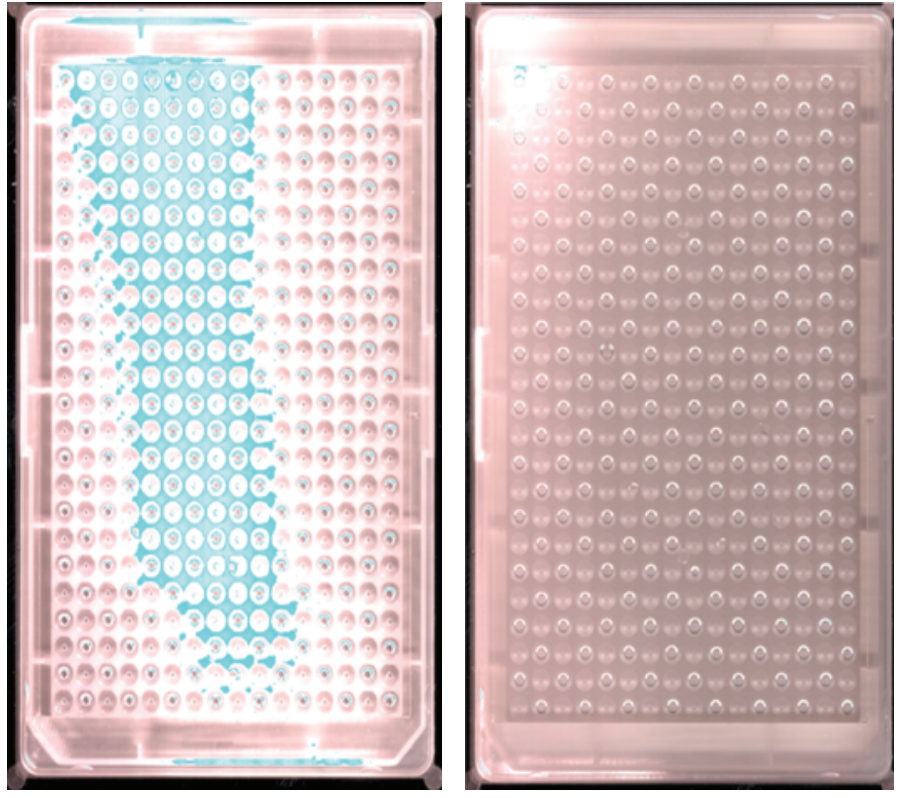

(a)

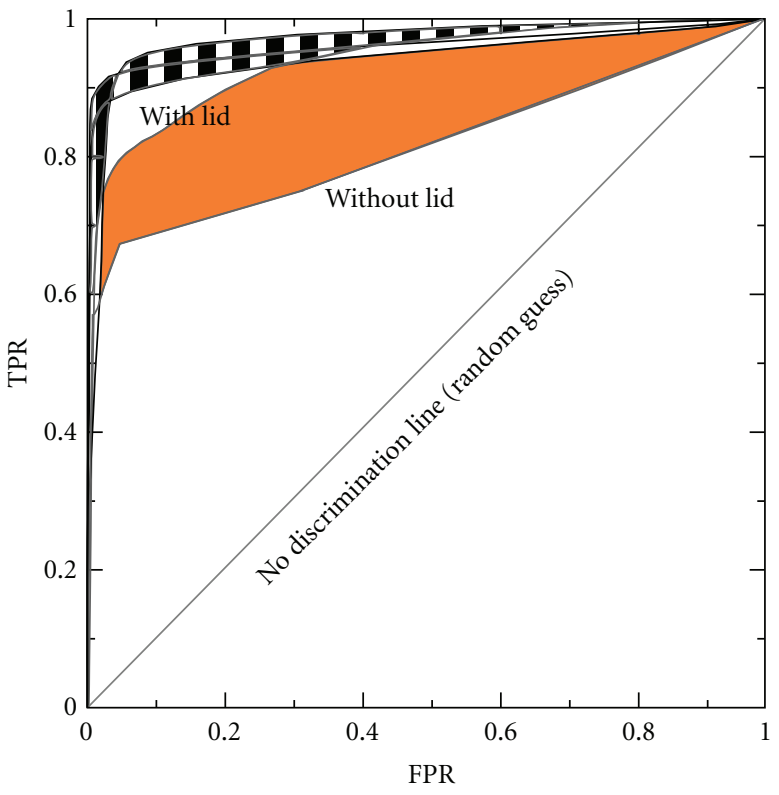

(b)

FIGURE 4: Overexposure without lid and with lid and results, V-PP-384 (CIS).

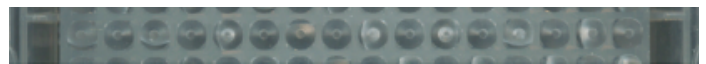

(a)

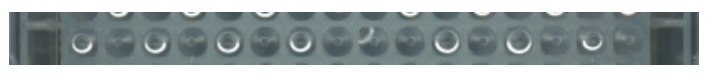

(b)

Figure 5: Influence of pipetting technique: (a) DMSO; (b) water.

into the middle of the well at a very high level of reproducibility. DMSO was pipetted using Tip Touch, which is not ideal for the substance; this increased the negative influence of droplets clinging to the well edges. Dosing DMSO also showed an increasing frequency of off-centre droplets at increasing volume, suggesting an operational range depending on substance. Figure 5 shows how a bad pipetting technique leads to uncentered drops clinging to the walls. As described in Section 3.2, this leads to a decrease in measurement accuracy. The accuracy decreases earlier and more rapidly for DMSO than it does for water using either sensor type. Optimised pipetting techniques play a major role in a reliable detection of pipetted droplets.

\section{Conclusion and Recommendations}

The system's functionality has been confirmed in this test sequence. Using a basic flatbed scanner simplifies application for pipetting monitoring and improves results while increasing efficiency compared to the camera solution used up to now.

The pipetting monitoring presented can be implemented using barcode readers based on CCD or CIS flatbed scanners that the lab may already have at its disposal. Efficacy with regard to sample volume and substance properties as well as robustness to ambient light was tested. Depending on lab lighting conditions, a covering may improve results. Further work should be done for the evaluation of all environmental variables influencing the sensor signals. Additionally error analysis of the developed system and comparison to classical methods will be done.

The results of the tests taken up to now have revealed a variety of possible improvements for the system; result quality showed heavy dependence on the algorithm parameters used, so automatic parameter optimisation should be used for the reliable detection of the best parameters. Additionally, this would enable the user to configure the device for new 
labware on his own, since all settings could then be found automatically.

In addition, adaptation to ambient light should be optimised to ensure that the system can be used without covering wherever possible. There is further room for improvement at high volume ranges or for badly positioned droplets; the potential for quantitative volume estimation from twodimensional droplet projections, fluid properties, and well shape warrants further investigation.

\section{Acknowledgment}

The authors wish to thank the Federal Ministry of Education and Research for the financial support of the Center for Life Science Automation (03ZIK 021).

\section{References}

[1] G. Porter, "Miniaturized assays magnify pipetting issues," American Biotechnology Laboratory, vol. 20, no. 2, p. 71, 2002.

[2] A. Balcombe, "On the level," Control and Instrumentation, vol. 26, no. 12, p. 19, 1994.

[3] M. J. Heim, Füllstandmesstechnik in der industriellen Praxis, VDI/VDE-Gesellschaft für Mess- und Automatisierungstechnik, 2002.

[4] S. Chakravarthy, R. Sharma, and R. Kasturi, "Noncontact level sensing technique using computer vision," IEEE Transactions on Instrumentation and Measurement, vol. 51, no. 2, pp. 353 361, 2002.

[5] G. J. Litt, "United States patent: 4824230—visualization device," U.S. patent 4824230, April 25, 1989.

[6] J. Pochert, "Liquid level detection in high-throughput screening applications," in International Biotechnology Laboratory, p. 44, 2000.

[7] D. Dossenbach and S. Jess, "Wenn der $\mu$-liter eine rolle spielt," in Mikrofluidik, p. 44, Oktober 2009.

[8] R. E. Schapire, "The boosting approach to machine learning: an overview," in Nonlinear Estimation and Classification, vol. 171, pp. 149-171, Springer, Berlin, Germany, 2003.

[9] H.-H Bothe, Neuro-Fuzzy-Methoden, Springer, Berlin, Germany, 1998.

[10] T. Fawcett, "An introduction to ROC analysis," Pattern Recognition Letters, vol. 27, no. 8, pp. 861-874, 2006. 


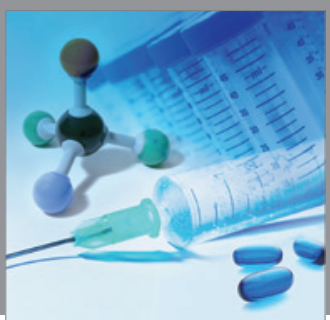

International Journal of

Medicinal Chemistry

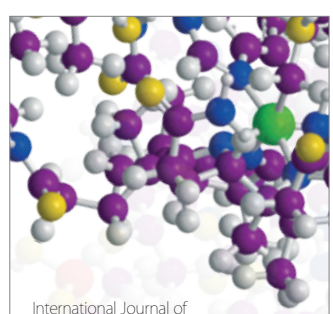

Carbohydrate Chemistry

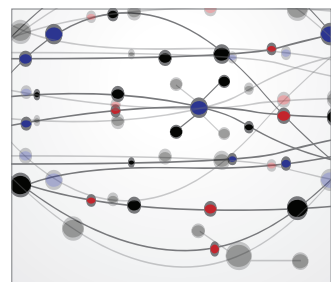

The Scientific World Journal
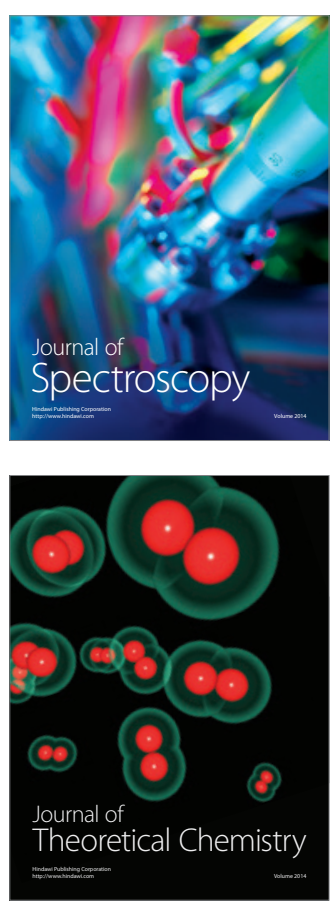
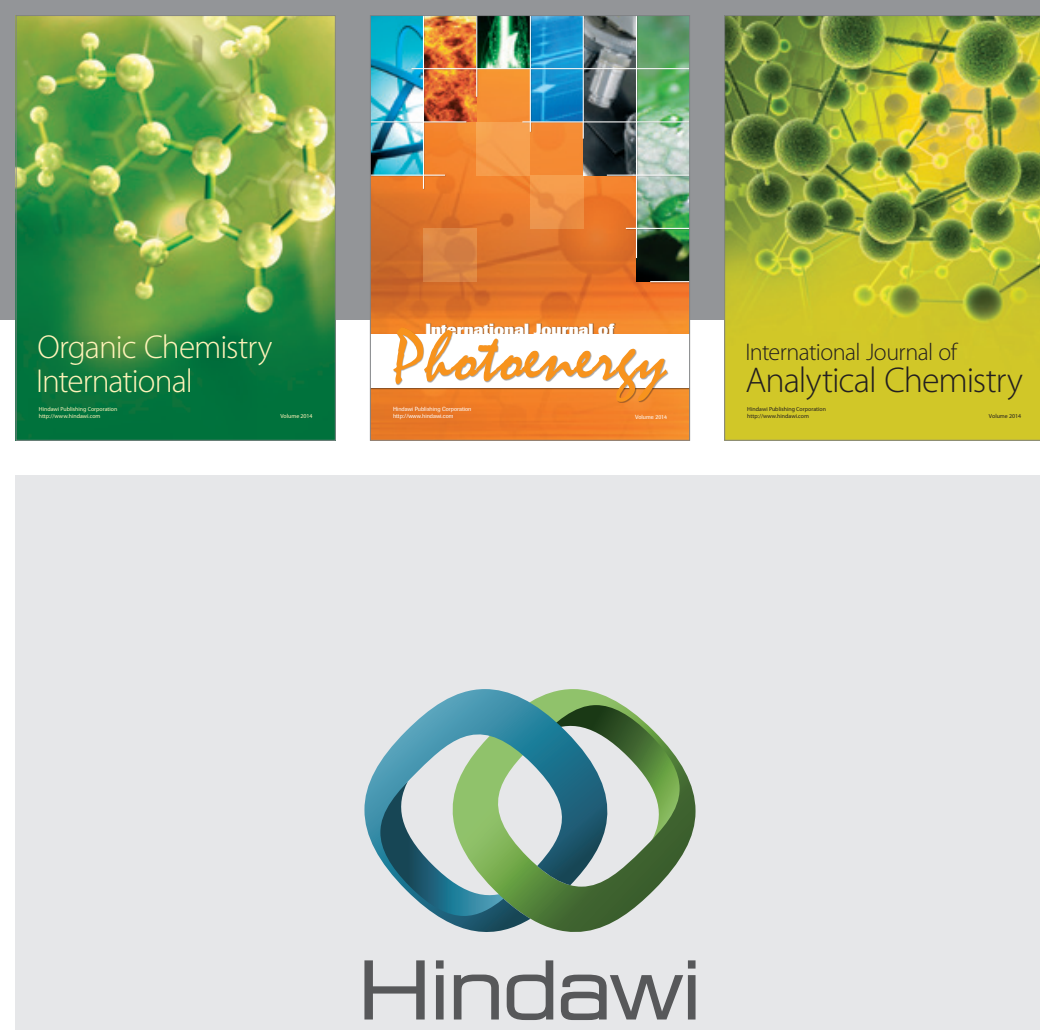

Submit your manuscripts at

http://www.hindawi.com
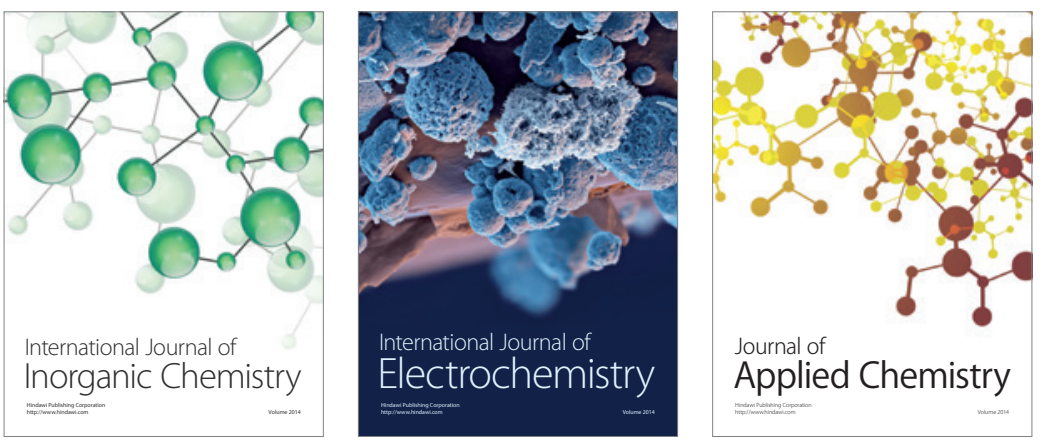

Journal of

Applied Chemistry
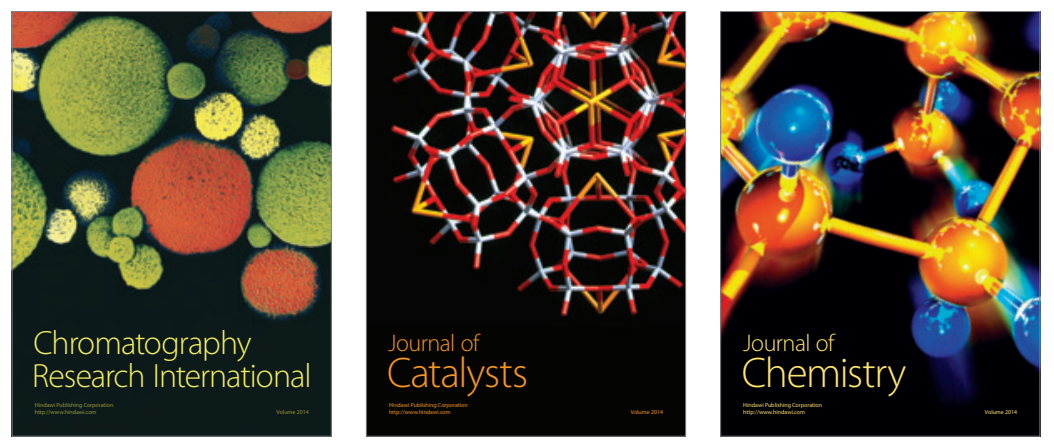
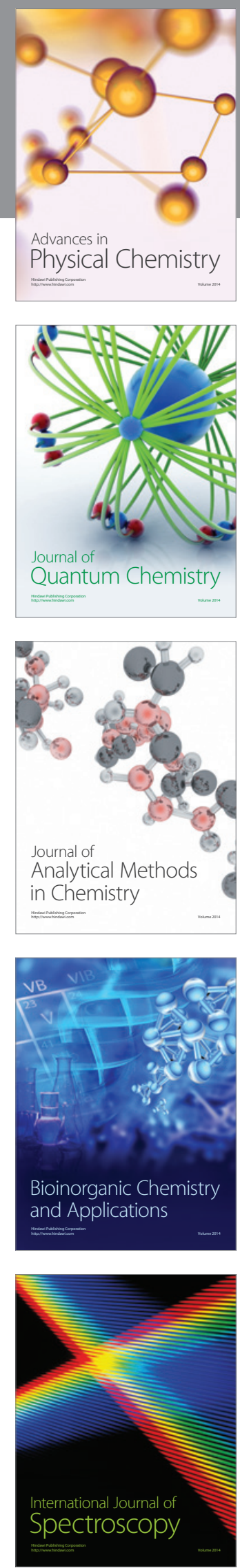